\title{
Identifying new topoisomerase II poison scaffolds by combining publicly available toxicity data and 2D/3D-based virtual screening
}

\author{
Anna Lovrics ${ }^{1}$, Veronika F. S. Pape ${ }^{1,2}$, Dániel Szisz ${ }^{3}$, Adrián Kalászi ${ }^{3}$, Petra Heffeter ${ }^{4}$, Csaba Magyar ${ }^{1}$ \\ and Gergely Szakács ${ }^{1,4^{*}}$ (i)
}

\begin{abstract}
Molecular descriptor (2D) and three dimensional (3D) shape based similarity methods are widely used in ligand based virtual drug design. In the present study pairwise structure comparisons among a set of 4858 DTP compounds tested in the NCl60 tumor cell line anticancer drug screen were computed using chemical hashed fingerprints and 3D molecule shapes to calculate 2D and 3D similarities, respectively. Additionally, pairwise biological activity similarities were calculated by correlating the 60 element vectors of pGI50 values corresponding to the cytotoxicity of the compounds across the NCI60 panel. Subsequently, we compared the power of 2D and 3D structural similarity metrics to predict the toxicity pattern of compounds. We found that while the positive predictive value and sensitivity of 3D and molecular descriptor based approaches to predict biological activity are similar, a subset of molecule pairs yielded contradictory results. By simultaneously requiring similarity of biological activities and 3D shapes, and dissimilarity of molecular descriptor based comparisons, we identify pairs of scaffold hopping candidates displaying characteristic core structural changes such as heteroatom/heterocycle change and ring closure. Attempts to discover scaffold hopping candidates of mitoxantrone recovered known Topoisomerase II (Top2) inhibitors, and also predicted new, previously unknown chemotypes possessing in vitro Top2 inhibitory activity.
\end{abstract}

Keywords: Mitoxantrone, Topoisomerase II poisons, NCI60 cell panel, Virtual screening, Scaffold hopping

\section{Introduction}

Drug resistance poses a serious challenge in the treatment of malignant diseases or bacterial infections, prompting the need for the development of new drugs. With the increased understanding of the genetic addictions, dependencies and vulnerabilities of cancer cells, target based approaches have yielded several successful treatment options, such as in the case of drugs developed against the epidermal growth factor receptor (reviewed in [1]). In addition, a significant number of novel FDA approved drugs across all therapeutic areas [2] and specifically in cancer [3] have been identified by phenotypic screens.

\footnotetext{
*Correspondence: szakacs.gergely@ttk.mta.hu

${ }^{4}$ Institute of Cancer Research and Comprehensive Cancer Center, Medical

University of Vienna, Borschkegasse 8a, 1090 Vienna, Austria

Full list of author information is available at the end of the article
}

Target and ligand based approaches are also widely used in virtual drug design. Opposed to target-based design, where drug binding to a known target is tested [4], ligand-based screening can be utilized also when the three dimensional (3D) structure of the target protein is not available [5]. Advances in computational techniques and hardware solutions have enabled in silico methods, in particular virtual screening, to accelerate lead identification and optimization [6].

In phenotypic screens, molecules are characterized by their biological function. The Developmental Therapeutics Program's (DTP) NCI60 panel is a collection of 60 human cancerous cell lines maintained by the National Cancer Institute (NCI). From 1990 more than 140,000 synthesized compounds and natural products were screened providing a vast repository of molecules for which both toxicity data and structural information are 
available [7]. Each drug-cell line pair can be characterized by the negative logarithm of the drug concentration that results in $50 \%$ growth inhibition of the given cell line (pGI50). Hence, each drug may be described by a 60 element vector, termed 'biological activity'. Several studies have found that biological activity is a strong predictor of the mechanism of action (MoA) of the compounds [8-13]. Moreover, compounds with previously unknown MoAs were correctly classified (see [7] and references therein), further supporting the use of toxicitybased biological activity patterns as a surrogate for MoA. In addition, by employing molecular descriptor-based methods, where molecules are converted to bit-strings such as chemical hashed fingerprint [14] or extended connectivity fingerprints [15], molecular structures can be analyzed with high speed and at a low computational cost. Methods for comparison of molecule shapes have also been developed to account for spatial features by maximizing the physical overlap of two molecules [16]. Whereas 3D methods have been successfully used to identify chemical leads with different scaffolds [17], 3D screening remains computationally expensive and it is challenging to find the biologically relevant active conformations of the compared molecules.

The relation of molecular descriptors to biological activity of the DTP compounds was extensively analyzed by Wallqvist et al. [18]. Here our aim was to characterize the relationship of different structural similarity measures to the cytotoxic patterns (i.e. biological activity) of the DTP compound set. Interestingly, we identified a set of compound pairs that were dissimilar in molecular descriptor based comparisons, but nevertheless displayed significant biological and 3D shape similarities. The same criteria would also define scaffold hopping pairs representing molecules of different core structures having comparable affinities to their molecular targets [19, 20]. To test this assumption, putative scaffold hopping analogues of the Top 2 poison anticancer agent mitoxantrone were analyzed by in silico docking calculations and in vitro decatenation assays.

\section{Results}

\section{Relation of structural similarity metrics and biological} activity of the DTP compounds

In order to assess the relation of structural similarities to biological activity, we calculated pairwise molecular descriptor similarities (chemical hashed fingerprint, CFP), 3D shape similarities (ROCS) and biological activity (BiolAct or BA) similarities among 4858 compounds analyzed by DTP's NCI60 screening project [7] (see Additional file 1: Fig. S1).

Additional file 1: Fig. S3 shows the distribution of 11,797,653 pairwise similarity values obtained from calculations assessing structural and biological overlaps of the molecules. The pairwise similarity values show a normal distribution, with different means and standard deviations for the structural metrics and the biological activity pattern. We assumed that high values represent significant similarities between the corresponding molecules. Indeed, the right threshold of the $95 \%$ confidence intervals (CI) of the bootstrapped distributions representing no-correlation are 0.30 and 0.22 for Pearson-correlation (BA similarity) and CFP similarity, respectively. Bootstrapped distribution could not be obtained for the 3D ROCS method, where the molecules are compared in a pairwise manner (see "Materials and methods").

In order to test how well either of the structural metrics predict biological activity, the positive predictive value (PPV) and sensitivity were calculated treating the structural metric similarity as the test and Pearson correlation as the true value. In this context, the positive predictive value defines the proportion of molecule pairs that simultaneously display structural and biological activity similarities to the total number of structurally similar molecule pairs (see Eq. (1)). Conversely, sensitivity is the number of molecule pairs that simultaneously display structural and biological activity similarities relative to the number of molecule pairs sharing similar biological activity (Eq. (2)). For any of the metrics, two compounds are considered similar, if their similarity score exceeds a chosen threshold value. Ideally, the selected threshold should warrant not only a high positive predictive value but also a high sensitivity. In our dataset, we find that while an increase of the threshold of a structural similarity metric increases the positive predictive value, it also results in a decrease of sensitivity.

Since the distributions of pairwise similarities differ for ROCS and CFP, we introduced percentiles as an independent variable to allow comparison of the $2 \mathrm{D}$ and $3 \mathrm{D}$ methods. Figure 1 shows that when percentiles are used to define thresholds, the positive predictive value and sensitivity curves are almost superimposable, suggesting that the overall effectivity of the molecular descriptor based and 3D metrics to predict biological activity is highly similar. The percentage of overlap among the distribution curves are $89 \%$ and $90 \%$ for positive predictive value and sensitivity, respectively. Interestingly, similarity in biological activity and molecular descriptor based structures does not necessarily imply 3D similarity. Likewise, there are molecule pairs that jointly satisfy $3 \mathrm{D}$ and biological activity similarities without showing any similarity according to the CFP metric (Fig 2).

We focused on molecule pairs showing high 3D similarity and a highly similar toxicity pattern-yet whose molecular descriptor based similarity did not suggest structural resemblance. This characteristic is reminiscent 

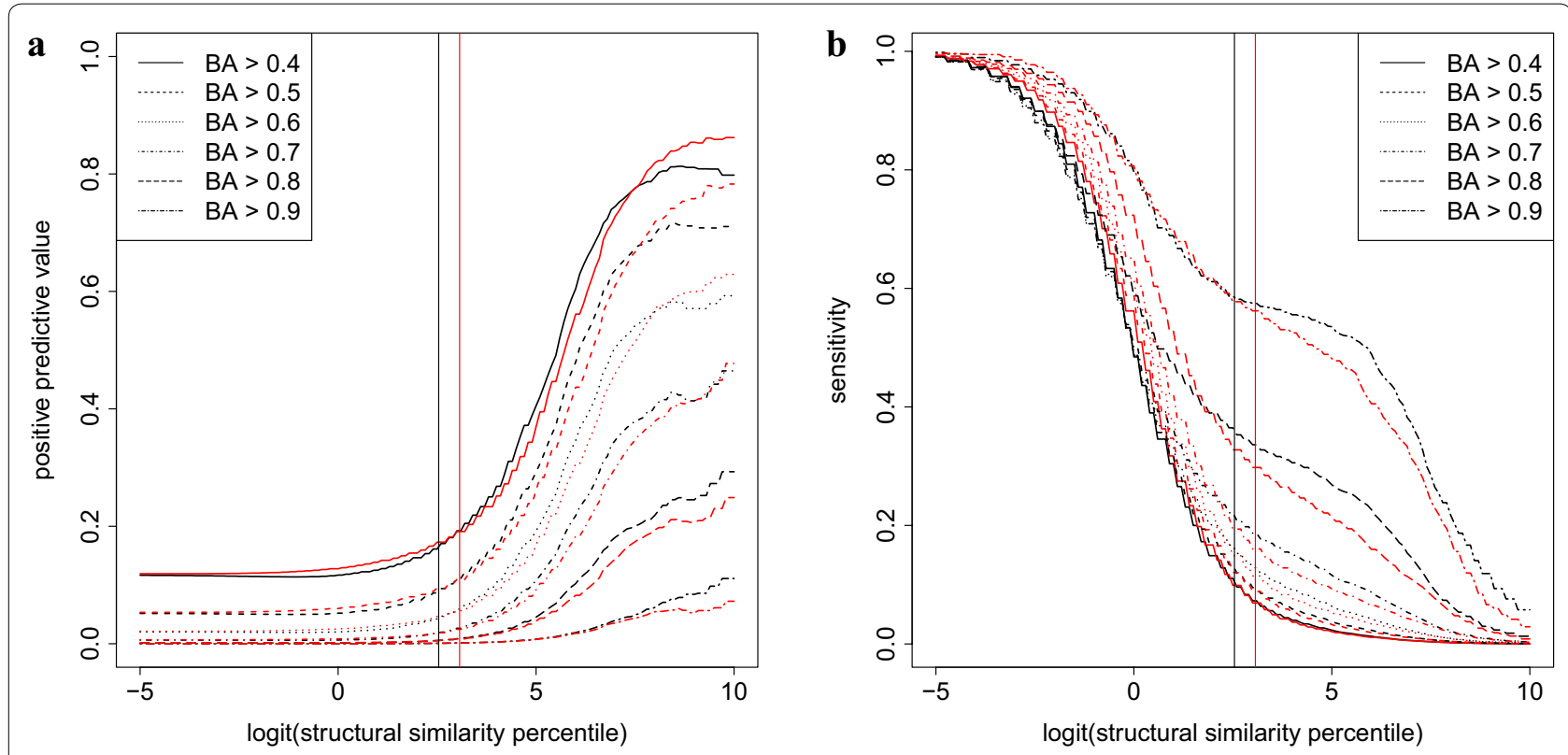

Fig. 1 Positive predictive value (a) and sensitivity (b) of ROCS (black) and CFP (red) similarities to predict biological activity (BA). Values are shown as a function of the percentiles of the number of molecule pairs displaying ROCS or CFP similarities. The thresholds of ROCS and CFP similarity values used for identification of scaffold hopping candidates of mitoxantrone are shown by black and red vertical lines, respectively

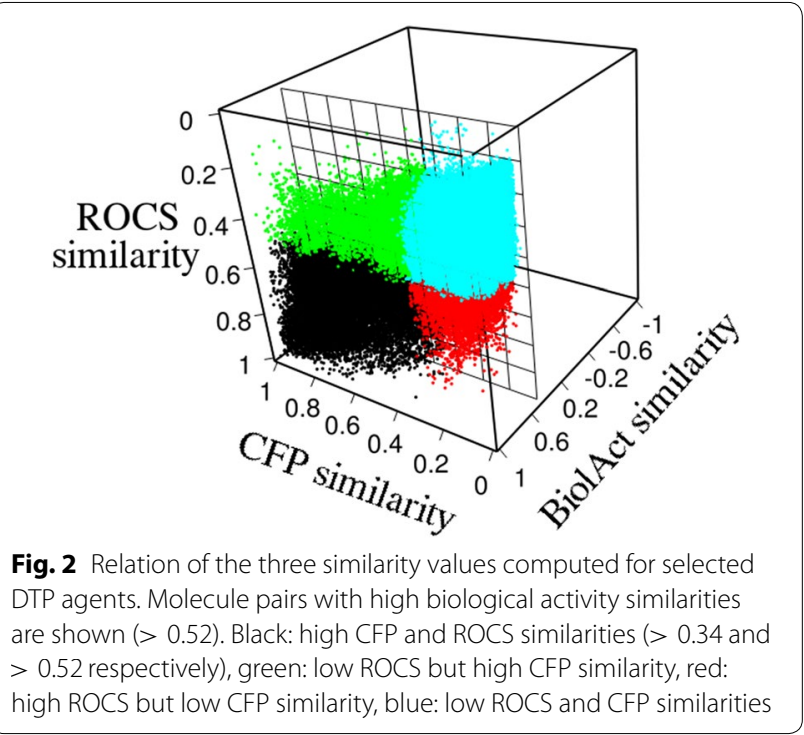

of 'scaffold hopping', i.e. the switch to a new chemotype without a compromise in biological activity.

\section{Identified scaffold hopping candidates of the Top2 poison mitoxantrone}

In search for scaffold hopping candidates we collected compounds showing high similarity to the $3 \mathrm{D}$ structure and the biological activity but low similarity to the molecular descriptor based fingerprint of FDA approved drugs among the 4858 structures analyzed in this study. A prominent group of highly diverse compounds was formed by compounds sharing 3D and biological similarity with the Top2 poison mitoxantrone. Within the subset of these agents, putative scaffold hopping candidates were identified based on dissimilarity of the molecular descriptor-based fingerprints (selection of similarity thresholds is detailed in "Materials and methods"). Briefly, candidates were chosen by considering the similarities of annotated Top2 poisons and inhibitors within the

\footnotetext{
(See figure on next page.)
}

Fig. 3 Top2 poisons in the set of 4858 DTP structures used in this study as obtained from Weinstein et al [59], supplemented by mechanism of action (MoA) information downloaded from the CellMiner website. The annotated Top2 poisons include ametantrone (NSC196473/NSC287513), the closest derivative of mitoxantrone, the anthracyclines daunorubicin (NSC83142), idarubicin (NSC256439), N,N-dibenzyldaunorubicin (NSC268242), epirubicin (NSC256942), doxorubicin (NSC123127), rubidazon (NSC164011) and valrubicin (NSC246131), as well as menogaril (NSC269148). Futhermore, piroxantrone (NSC349174), bisantrene (NSC337766), amsacrine (NSC141549/NSC154948/NSC156303/NSC249992), ellipticiniums (NSC351710, NSC638066) and podophyllin derivatives (etoposide: NSC141540, teniposide: NSC122819), as well as dexrazoxane (NSC169780) 


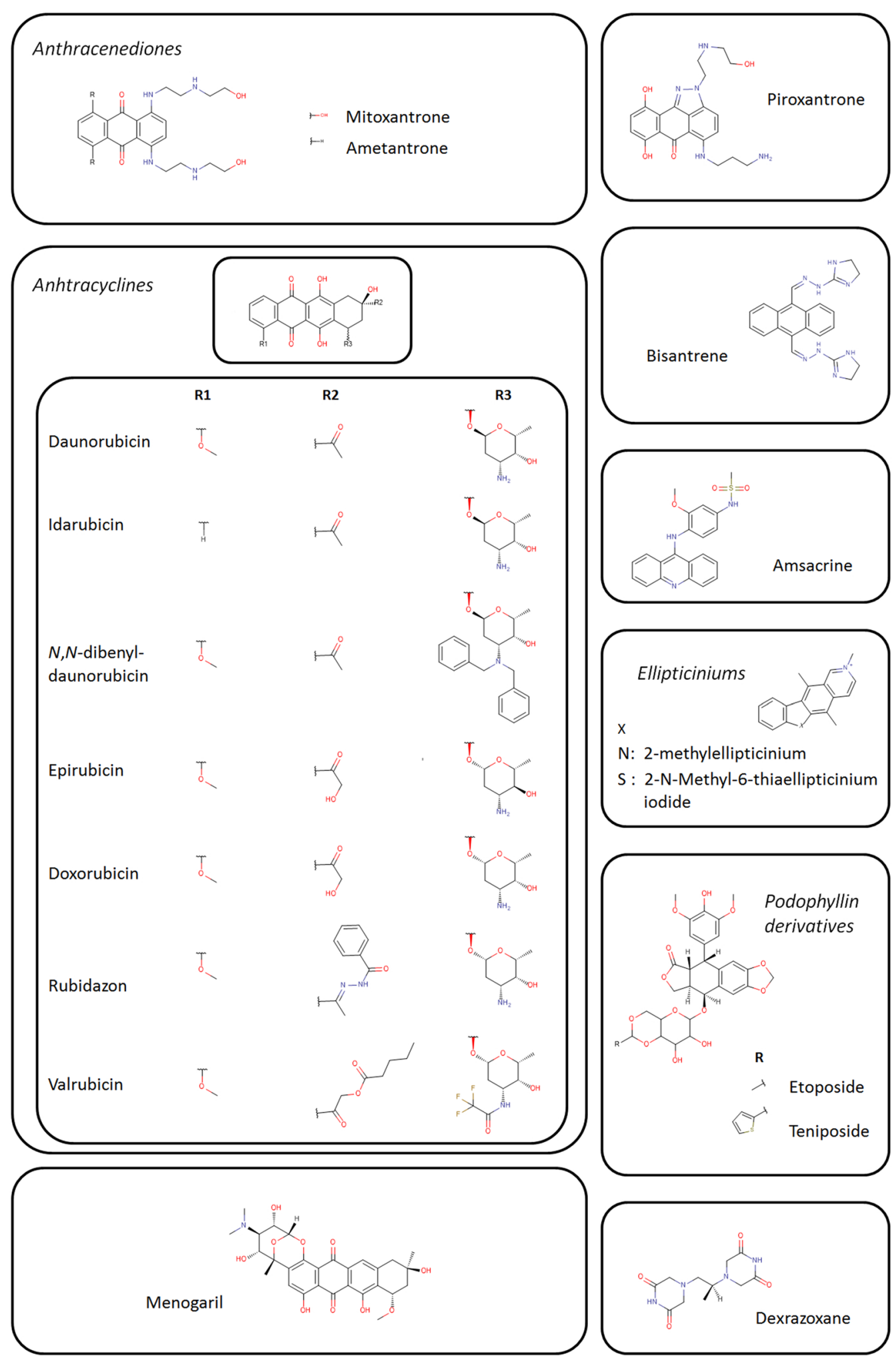




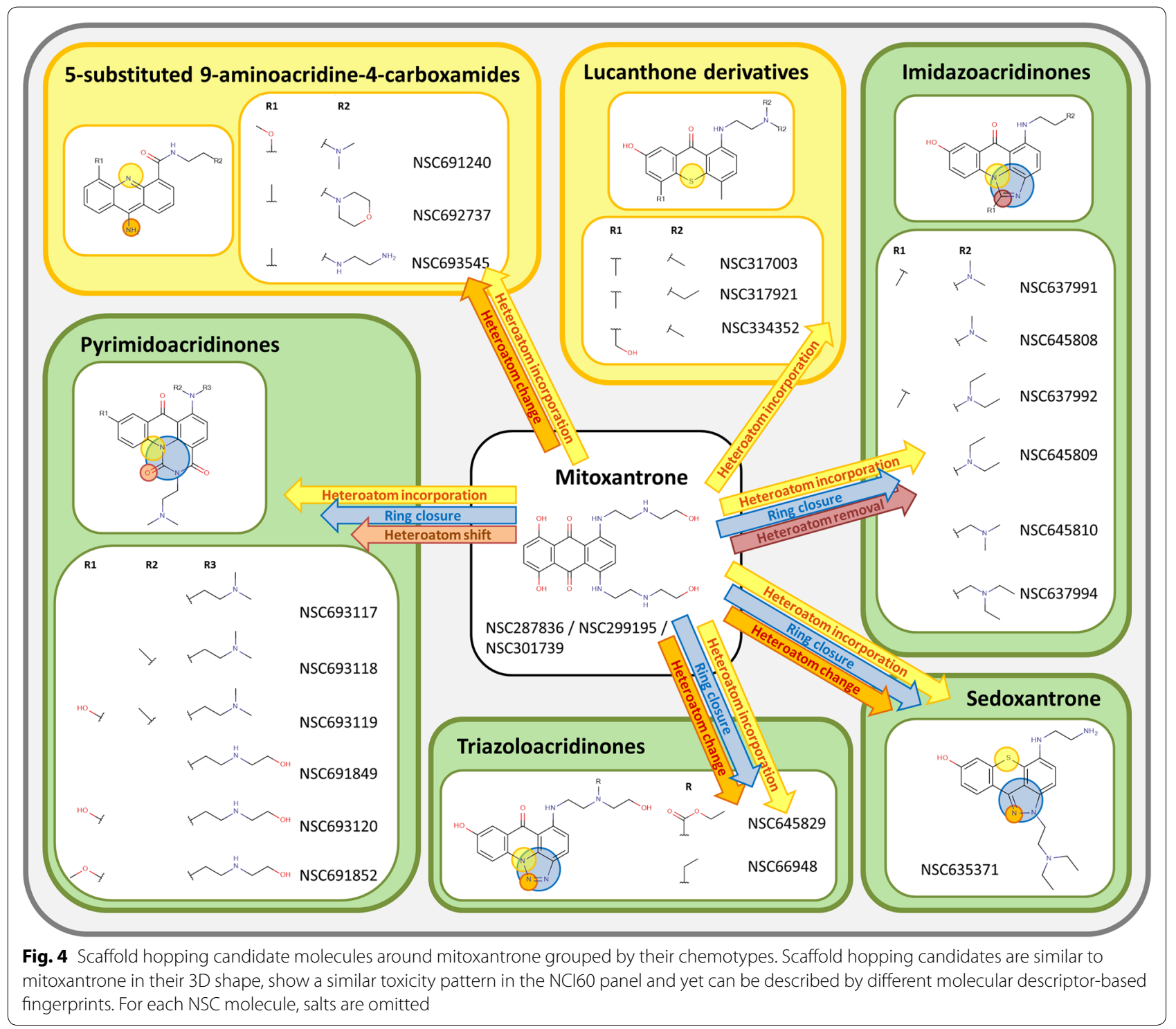

compounds set (Fig. 3) to mitoxantrone. As presented in Fig. 1, the chosen thresholds (BA: 0.53; ROCS: 0.51; CFP: 0.32 ) represent a compromise between reasonable sensitivity and PPV values for the whole dataset. Within the subset of agents showing high $3 \mathrm{D}$ and biological similarity to mitoxantrone (BA $\geq 0.54$, ROCS $\geq 0.51$ ), putative scaffold hopping candidates were identified based on dissimilarity of the molecular descriptor-based fingerprints (CFP < 0.32). This approach identified 20 scaffold hopping candidates, representing six distinct chemotypes. As expected based on the similarity criteria, the structure of the compounds show characteristic differences (Fig. 4). SMILES of the scaffold hopping candidates are listed in Additional file S1.
Given the similar biological activity of the compounds shown in Fig. 4, we expected that their MoA relied on Top2 inhibition. Whereas none of the scaffold hopping candidates is annotated as a Top2 poison, some agents, including sedoxantrone (NSC635371), three of the six imidazoacridinone derivatives (NSC637992, NSC645809, NSC645810) [21] and the 5-substituted 9-aminoacridine-4-carboxamides [22] could be linked to Top 2 poisoning by literature search. Apart from these compounds, the remaining imidazoacridinone derivatives (NSC637991, NSC637994, NSC645808), the lucanthone derivatives (NSC317003, NSC317921, NSC334352), the triazoloacridinones (NSC645829, NSC699148) and the pyrimidoacridines (NSC693117, NSC693118, NSC693119, NSC693120, NSC691849, 
NSC691852) represent novel scaffold hopping candidates of mitoxantrone. Based on the range of the pGI50 values measured in the NCI60 cell panel, the activities of these compounds are in the range of the toxicity of mitoxantrone, but their potential to inhibit Top2 has not been investigated so far.

\section{Verification of the MoA of the scaffold hopping candidates}

Similar toxicity patterns (i.e. biological activity) along with similar 3D structures suggest that-similarly to mitoxantrone-the compounds shown in Fig. 4 kill cells by binding to the active site of Top2. To verify this proposition, binding of the scaffold hopping candidates to the Top2-DNA adduct was quantified by in silico docking calculations using protein coordinates reported by $\mathrm{Wu}$ et al. [23, 24]. In addition to the scaffold hopping candidates (Fig. 4) and the DUDE-E generated decoy structures, simulations were run for a 3D shape similar but biologically distinct ('3D decoy'), and a biologically similar but 3D shape distinct ('biological decoy') set of molecules. Docking scores obtained for individual molecules are displayed in Additional file 1: Tables S5-S7. Since this search recovered relatively few decoy structures, the search was extended to include similarities in the context of the scores obtained for any of the published Top2 ligands mitoxantrone, ametantrone, amsacrine and etoposide [23, 24]. While five additional putative scaffold hopping candidates arose (Additional file 1: Fig. S4 and Table S3), the overall distribution of the scaffold hopping docking scores did not change (Fig. 5). Docking scores and ranks obtained for the putative scaffold hopping analogues of mitoxantrone are shown in Table 1 and Additional file 1: Table S4, respectively; scores for the '3D decoy' and 'biological decoy' sets are shown in Additional file 1: Tables S5, S6. As displayed in Fig. 5, scaffold hopping candidates exhibit a significantly lower docking score than any of the decoy sets, suggesting that the toxic activity of these compounds relies on binding to the Top2-DNA adduct. Mitoxantrone and different chemotypes of scaffold hopping molecules overlap well and share the same binding site, only the longer side-chain of mitoxantrone is involved in additional interactions with the receptor structure (Fig. 6).

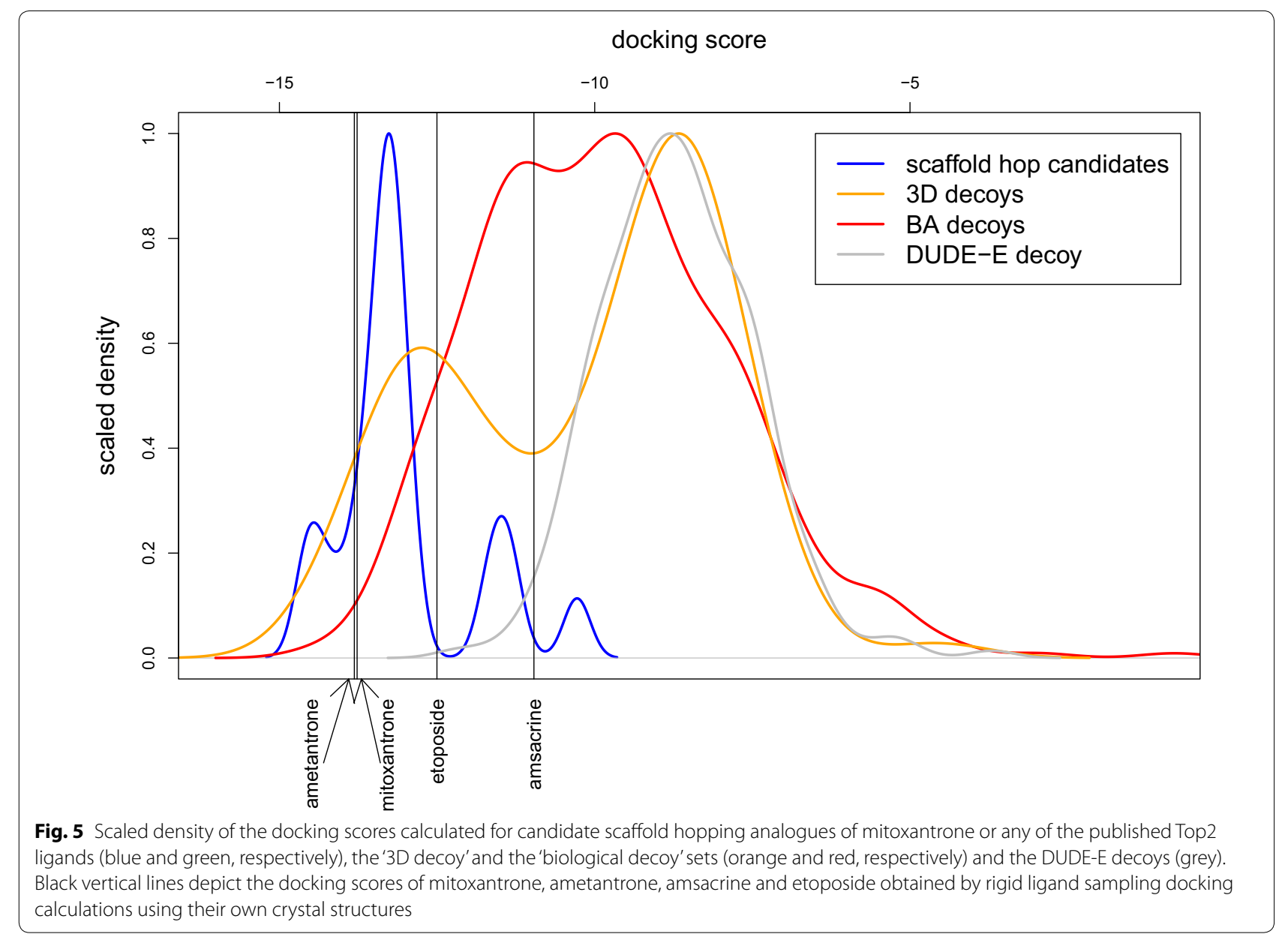


Table 1 Biological, ROCS and 2D similarities compared to mitoxantrone

\begin{tabular}{llllll}
\hline Chemotype & NSC & BiolAct & ROCS & CFP & Docking \\
\hline Mitoxantrone & 301739 & & & & -13.45 \\
Lucanthone derivatives & 317003 & 0.73 & 0.55 & 0.28 & -11.31 \\
& 317921 & 0.66 & 0.55 & 0.29 & -11.47 \\
& 334352 & 0.74 & 0.55 & 0.28 & -11.67 \\
Triazoloacridinones & 645829 & 0.56 & 0.62 & 0.27 & -13.41 \\
& 699148 & 0.58 & 0.54 & 0.26 & -10.28 \\
Pyrimidoacridines & 693117 & 0.67 & 0.58 & 0.22 & -13.19 \\
& 693118 & 0.68 & 0.53 & 0.22 & -14.48 \\
& 693119 & 0.70 & 0.57 & 0.25 & -13.53 \\
& 693120 & 0.70 & 0.63 & 0.26 & -14.57 \\
& 691849 & 0.79 & 0.65 & 0.24 & -13.81 \\
Sedoxantrone & 691852 & 0.54 & 0.63 & 0.26 & -14.17 \\
Imidazoacridinones & 635371 & 0.67 & 0.60 & 0.24 & -13.32 \\
& 637991 & 0.60 & 0.53 & 0.27 & -13.66 \\
& 637992 & 0.66 & 0.53 & 0.28 & -13.25 \\
4 Carboxamides & 637994 & 0.76 & 0.52 & 0.26 & -13.25 \\
\hline idine & 645808 & 0.76 & 0.53 & 0.28 & -13.33 \\
& 645809 & 0.76 & 0.53 & 0.29 & -13.00 \\
& 645810 & 0.84 & 0.52 & 0.26 & -13.27 \\
& 691240 & 0.68 & 0.52 & 0.26 & -13.08 \\
& & & & & \\
& 69345 & 0.63 & 0.52 & 0.25 & -12.95 \\
\hline
\end{tabular}

Docking scores of scaffold hopping candidates
Finally, we tested the actual Top2 poisoning potential of mitoxantrone analogs and a '3D decoy' (Additional file 1: Fig S7) molecule that were made available by DTP in in vitro decatenation assays. The obtained IC50 value of mitoxantrone is in line with the published literature [25], while for imidazoacridinones, only yeast Top 2 assay results were previously available [21]. As shown in Fig 7, the three scaffold hopping analogs representing the imidazoacridinones, lucanthones and the aminoacridines exhibited significant Top2 inhibitory activities (summarized in Table 2), while NSC660839 showed no inhibition, despite the fact, that its docking score (depicted in table S5) was comparable to that of etoposide, a ligand co-crystallized with Top2 [23].

\section{Discussion}

The DTP's NCI60 is an information rich resource which has provided valuable insights into the MoA of the compounds as well as mechanisms of cellular sensitivity and resistance [7]. Earlier studies have established the relation of drug toxicity profiles to structural clusters and distinct modes of action (reviewed in [7]). In particular, molecular descriptor-based (2D) structural similarity was extensively studied by Wallqvist et al., who quantified the effect of structural changes on biological activity within the DTP molecule set [18]. Our first goal was to compare the power of 2D and 3D structural similarity metrics to predict biological similarity. We used the measures of positive predictive power and sensitivity to assess whether 3D shape based similarities would perform

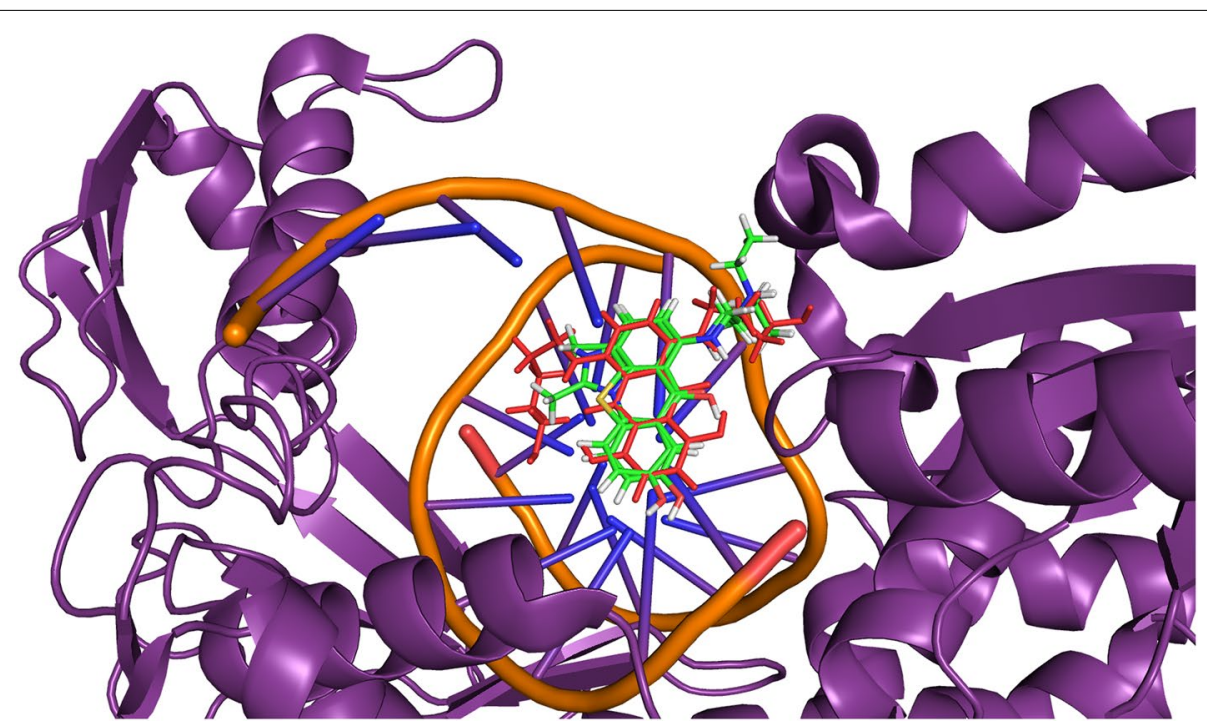

Fig. 6 The structure of human Top2 $\beta$ is shown with purple color in cartoon, while ligands are shown in stick representation. Co-crystallized structure of mitoxantrone is marked in red color, while poses of NSC317921 and NSC637992 obtained by Glide SP docking calculations are colored using atom types 


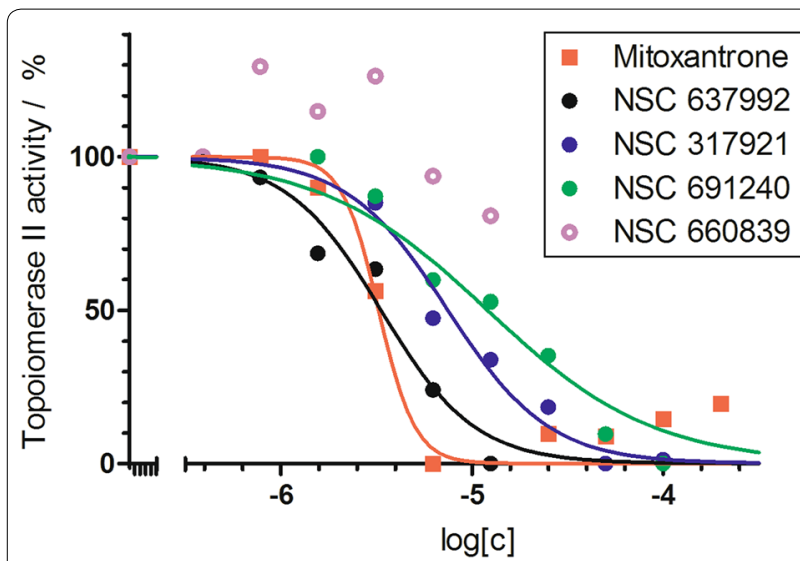

Fig. 7 Effect of the test compounds on Top2-mediated decatenation of kinetoplast DNA (KDNA). Dose-dependent poisoning was calculated from DNA samples separated by gel electrophoresis. Symbols represent experimental data, continuous curves are fitted lines. Red: mitoxantrone, black: NSC637992, blue: NSC317921, green: NSC691240, purple: NSC660839. Filled and open circles represent scaffold hopping and decoy molecules, respectively. A representative gel photo is shown in Additional file 1: Fig S5

Table 2 Calculated Top2 poisoning IC50 values of test compounds

\begin{tabular}{lll}
\hline NSC & $\mathbf{I C 5 0}(\boldsymbol{\mu M})$ & $\mathbf{9 5 \%} \mathrm{Cl}(\boldsymbol{\mu M})$ \\
\hline Mitoxantrone & 3.3 & $2.6-4.1$ \\
637992 & 3.3 & $2.5-4.6$ \\
317921 & 7.5 & $5.9-9.6$ \\
691240 & 12.1 & $9.0-16.3$ \\
660839 & $>100$ & $\mathrm{NA}$ \\
\hline
\end{tabular}

better than molecular descriptor based methods at predicting the similarities of biological activities. In virtual screening campaigns, the positive predictive value can be interpreted as the fraction of truly biologically similar molecules among the compounds obtained by structure similarity search, whereas sensitivity defines the fraction of compounds returned by the structure search among all of the biologically similar molecules. The 2D CFP and 3D ROCS metrics were similar in their ability to predict biological function (Fig. 1), a result in accordance with findings of benchmark studies that have not detected clear evidence of superiority of 3D methods [26-29].

Figure 2 shows the relation of structural and biological similarity metrics. As expected, a large number of molecules are similar in structural and biological aspects. More interestingly, we identified biologically similar molecule pairs for which only either of the $2 \mathrm{D}$ or the $3 \mathrm{D}$ structural metrics proved to be similar. For example, we found compound pairs that are dissimilar according to the 3D similarity measure, but nevertheless show high similarity in their 2D structures and biological activities (Fig. 2, green). This can occur if a relatively large structural moiety appears once in one of the molecules and multiple times in the other molecule. Naturally, in this case, 3D shape similarity is not observed. On the other hand, such molecule pairs may highlight substructures that are essential for biological activity. Molecule pairs that are structurally dissimilar based on both $3 \mathrm{D}$ and 2D comparisons despite their biological activity similarities are displayed in blue. Structural dissimilarity despite biological similarity is a common phenomenon, examples include structurally diverse substrates of transporters [30]; range of ligands of the same protein target that may adopt multiple conformations or simply ligands of different protein targets that belong to the same pathway. Finally, there are agent pairs that are dissimilar in 2D despite high 3D and biological similarities. We hypothesized that this subset would be enriched in scaffold hopping candidates.

An important goal in the initial phase of drug discovery is to increase the quality of drug candidates [31]. Scaffold or lead hopping, defined by similar biological activity of different molecular backbones, could contribute to this aim. Despite their different core structures, scaffold hopping molecule pairs show comparable affinities to their molecular targets [19]. Scaffold hopping analogues may exhibit better physicochemical and pharmacokinetic properties while retaining the original potency, thus providing a new direction for further optimization. Scaffold hopping has been employed to discover novel compounds for drug development in the case of a variety of diseases, including finding scaffold hopping analogues of natural compounds [32]. Overall, finding scaffold hopping variants of active molecules is an integral part of virtual screening in the drug discovery pipeline [5]. While a wide variety of similarity search approaches exist to identify structural analogues to a lead compound, to our best knowledge, there is not a single, commonly accepted in silico method to identify scaffold hopping molecules. Here, we propose a method to identify biologically similar molecules to a query compound that are distinct in their core structure. While an experienced medicinal chemist may readily identify the remote similarity to mitoxantrone of the structures displayed in Fig. 7, it would be impossible to visually screen thousands of compounds. Also, the MoA of these compounds may differ from that of the original active molecule. We introduce similarity of biological activity as an additional criterion to obtain molecule pairs whose MoA is expected to be identical despite differences in their scaffolds.

We tested the validity of our approach by performing in silico and in vitro experiments with scaffold hopping candidates of the Top2 inhibitor mitoxantrone. Since we 
introduced 2D dissimilarity to mitoxantrone as a condition, the scaffold hopping candidates listed in Table 4 represent different chemotypes. Next to known Top2 poisons, including anthracyclines, structures were recovered that were linked to Top2 poisoning in the literature [21, 22], but some of the analogs represented novel chemotypes for Top2 poisoning. In comparison to mitoxantrone, these structures have additional heteroatoms incorporated in their rings, as in the case of lucanthone derivatives; or a heteroatom incorporation with an additional heteroatom change, as in the case of the 5-substituted-9-aminoacridine 4 carboxamides. A further ring closure can be observed in the triazoloacridinones and in sedoxantrone, which show high similarity to the known Top2 poison piroxantrone (see Fig. 3). Imidazoacridinones are derivatives of mitoxantrone, in which a heteratom is incorporated into a ring, another one is removed and additionally a ring is formed. Pyrimidoacridinones differ from mitoxantrone by a heteroatom incorporation, a ring closure and a shift of another heteroatom. A remote similarity of the scaffold hopping candidates can also be seen to amsacrine, which is a known Top2 poison (Fig. 3). However, these molecules still represent new chemotypes among Top2 poisons.

As the principal MoA of mitoxantrone is Top2 poisoning, activity of the scaffold hopping set could be verified by in silico docking and in vitro decatenation assays.

Scaffold hopping candidates of mitoxantrone obtained similar docking scores as the published Top2 ligands mitoxantrone and ametantrone, and notably, better docking scores than etoposide and amsacrine and the majority of known Top2 poisons. By defining biological and structural decoy sets (agents where only either the biological activity or 3D structural similarity to a published Top2 ligand is above the chosen thresholds), we found that both properties were required to obtain good docking scores. We note that some of the biological decoy molecules possess docking scores as good as the scaffold hopping candidates, and hence can be thought of false negatives. However, on the one hand, it is expected that some structurally diverse molecules would also fit well to the Top2 binding site. Still, the majority of structurally distinct biological decoys display a worse docking score. Taken together, some scaffold hopping candidates might have been lost by applying the methodology presented in this paper, but the number of false positives was also reduced significantly. On the other hand, ROCS similarity was found to perform better than docking in search for active molecules [5], and hence it could also be hypothesized, that in some cases, the docking calculation produces false positive results.

In order to test whether using different thresholds for the similarity metrics would provide better docking score separation between the scaffold hopping candidates and the biological decoy molecules, Fig. 5 was replotted using stricter (higher BiolAct and ROCS and lower CFP) and more lenient (lower BiolAct and ROCS and higher CFP) similarity threshold values. While at more stringent threshold values the separation of scaffold hopping candidates and decoy molecules is more pronounced, there remains a subset of '3D decoy' molecules displaying good docking scores (Additional file 1: Fig. S6).

Scaffold hopping candidates made available by DTP were also evaluated in in vitro decatenation assays, which demonstrated that NSC637992, NSC317921 and NSC691240 are efficient Top2 poisons, while NSC660839, a '3D decoy' molecule does not show inhibitory power despite the fact that it obtained a good docking score.

The list of 4858 DTP compounds used in this study and their pairwise structural and biological similarities are available in Additional files S2 and S3 respectively. Based on this dataset, scaffold-hopping candidates of any arbitrary agent may be obtained. As a further example, scaffold hopping candidates of camptothecin (NSC94600), a Topoisomerase I (Top1) inhibitor were also collected [33]. Additional file 1: Figs. S8 and S9 depict known Top1 inhibitor scaffolds and the scaffold hopping candidates, respectively. Threshold values to obtain scaffold hopping analogues around camptothecin were obtained similarly to those for mitoxantrone (see "Materials and methods"), by comparing the biological activity to known Top1 inhibitors. However, this approach could not be followed to identify scaffold hopping analogues of podofilox (NSC24818). Podofilox targets Eg5, a human kinesin involved in the formation of the bipolar spindle [34], and as such, could be categorised as tubulin affecting antimitotic. Still, biological activities of DTP compounds annotated as tubulin affecting agents display such a wide variety, that the minimum of the biological activity similarities of these molecules to podofilox represent random correlation among the set of 4858 DTP agents. Hence, in the case of podofilox, putative scaffold hopping candidates were selected using similarity thresholds corresponding to the 90th percentile of all pairwise similarities for each metric. Additional file 1: Figs. S10 and S11 represent the structure of podofilox and its putative scaffold hopping analogs, respectively.

The presented methodology can be applied to any set of molecules whose biological activity can be quantitatively compared in a pairwise manner. For this purpose, the DTP NCI60 database serves as a unique resource, but CMap [35], SIDER [36, 37] or chemogenomic databases [38] or the calculated ADMET properties [39] could also provide the starting point to search for candidate scaffold hopping molecule pairs. Similarly, different structural 
similarity metrics could be employed. For the molecular descriptor similarity calculations, ChemAxon's CFP [40] was chosen as a well-established, widely used measure, however other similar metrics (e.g. the Extended Connectivity Fingerprint) may also be applied. OpenEye's ROCS was chosen for 3D calculations as the most widely used and tested method [17], however, other alternatives exist (e.g. ChemAxon's Screen3D [41], pharmacophore fingerprints using either fuzzy molecular representations [42] or combined with ranking, voting, and consensus scoring [43]). It has to be noted that parametrization of the chosen metric, the similarity calculation method and the selection of the thresholds may also influence the outcome of similarity estimations. For instance, in the ROCS calculations, the most similar conformations among tested molecule pairs were used to assign the similarity score of the compounds. If the biologically active conformations are not known or in case of a general database search this might be the method of choice, however, the presented in silico application to find scaffold hopping analogues of a main compound should always be tailored to the actual task.

\section{Conclusion}

We have introduced a method to generate scaffold hopping molecule pair candidates by simultaneously calculating biological activity, 3D shape and molecular descriptor based similarities. Scaffold hopping candidates of mitoxantrone displayed typical examples of core structural changes such as heteroatom/heterocycle change and ring closure. The method was able to recover known Top2 inhibitors and additionally predicted new, previously unknown chemotypes possessing in vitro Top2 inhibitory activity.

\section{Materials and methods}

\section{Description and curation of DTP toxicity data} and molecular structures

Structural information and pGI50 values were downloaded from the DTP websites (https://wiki.nci.nih. gov/display/NCIDTPdata/Chemical+Data and https:// wiki.nci.nih.gov/display/NCIDTPdata/NCI-60+Growt h+Inhibition+Data: GI50 Data (Sept 2014) respectively). Chemical and biological data curation inspired by [44, 45] is detailed in Additional file 1: Text and Fig. S1.

Briefly, for downloaded agents, pGI50 values were available for some or all of the NCI60 cell lines. Missing values or GI50 values equal to tested minimal or maximal drug concentrations were replaced by 'NA'. Compounds with more than 30 'NA' values were omitted; the remaining set was filtered to retain compounds showing variable toxicity (standard deviation of pGI50 values $\geq 0.4$ )
[46, 47]. Correlations of pGI50 values across the cell lines of compounds measured multiple times were generally good as shown in Additional file 1: Fig. S2, demonstrating the reliability of the DTP dataset.

Biological curation was followed by chemical standardization: non-covalently bound fragments were removed from the structures using ChemAxon's Standardizer [48]; if these fragments were physiologically relevant ions (i.e. $\mathrm{Na}^{+}, \mathrm{Cl}^{-}, \mathrm{SO}_{4}{ }^{2-}$ ), then the desalted compound was retained, otherwise the agent was omitted. Additional structures were either fixed when possible, or removed based on problems related to valence, formal charge and stereochemistry as defined by Structure Checker (ChemAxon) [49]. Inorganics and metal-containing molecules were also removed using an in-house script. Remaining agents were dearomatized and nitro groups were transformed into customized representations by ChemAxon's Standardizer [48]. The final structures were tested by both ChemAxon's Structure Checker [49] and OpenEye's OMEGA [50]. Since the DTP structure set only contains 2D structure information, compounds with undefined stereocenters were kept-even though this added some uncertainty to the 3D shape-based similarity calculations.

The final set contained 4858 unique structures after removal of desalted molecules that represented duplicate or triplicate structures as obtained by ChemAxon's duplicate search [51]. In the case of low biological activity correlation among duplicate structures, the agents were omitted, while in the case of high correlation of toxicity values of the duplicate structures, pGI50 values were averaged resulting in a final set of 4858 unique structures. The workflow of molecule selection is depicted in Additional file 1: Fig. S1.

\section{Similarity calculations}

Pearson correlation was used to calculate the similarity between biological activites (drug toxicity profile vectors) of the DTP compounds, handling missing pGI50 values by casewise detection. In total, 11,797,653 pairwise similarity values were obtained. ROCS (OpenEye Scientific Software, Santa Fe, NM) was used to calculate 3D shape-based overlaps $[52,53]$. For calculating 3D similarity, each compound was expanded into a set of 3D conformers using OpenEye's OMEGA. For each molecule, a maximum number of 200 conformers were generated and assembled in an energy sorted order [50,54]. When a molecule contained undefined stereocenters, random stereocenters were defined during conformer generation. For each pair of structures, 3D overlaps between all of the the available conformers were calculated using ROCS [52, 53], by applying the '-subrocs' option (starting the search at heavy atoms of the larger molecule) without further optimization. The highest similarity score was accepted 
as the 'ROCS' similarity between the selected pair of molecules. To obtain structural similarity based on molecular descriptors, ChemAxon's chemical fingerprint (CFP) [40] was utilized using the default parameters and the Tanimoto metric as the similarity measure. Similarity was calculated between each of the 4858 individual structures, resulting in 11,797,653 pairwise similarity values. The exact commands and parameters used for the molecular similarity methods are shown in Additional file 1: Text, and Fig. S1 summarizes the similarity calculations.

Motivated by the work of Wallqvist et al. [18], we define

$$
\begin{aligned}
& F\left(s=s_{T} \mid \rho=\rho_{T}\right)=\frac{N\left(s \geq s_{T} ; \rho \geq \rho_{T}\right)}{N\left(s \geq s_{T} ; \rho \geq-1\right)}, \\
& F\left(\rho=\rho_{T} \mid s=s_{T}\right)=\frac{N\left(s \geq s_{T} ; \rho \geq \rho_{T}\right)}{N\left(s \geq 0 ; \rho \geq \rho_{T}\right)},
\end{aligned}
$$

where $N\left(s \geq s_{T} ; \rho \geq \rho_{T}\right)$ represent the number of molecule pairs for which the value of the structural similarity metric $(s)$ is larger or equal than the requested threshold $s_{T}$ and the value of the Pearson correlation $(\rho)$ is simultaneously larger or equal than the requested threshold $\rho_{T}$.

The measures in Eqs. (1-2) quantify how well structural similarities predict biological response. Specifically, $F\left(s=s_{T} \mid \rho=\rho_{T}\right)$ expresses the fraction of molecule pairs with at least $s_{T}$ structural similarities that also show a minimum of $\rho_{T}$ biological activity similarities, i.e. the positive predictive value. Similarly, $F\left(\rho=\rho_{T} \mid s=s_{T}\right)$ indicates the fraction of molecule pairs with at least $\rho_{T}$ biological activity similarities that also share a minimum of $s_{T}$ structural similarities, i.e. the sensitivity. These measures were calculated for both structural metrics (ROCS and CFP) among the selected DTP agents.

\section{Similarity threshold selection to mitoxantrone}

Selected cut offs between agent pairs considered similar or dissimilar can be tailored to the needs of the actual study, considering expected increase and decrease of false positives and negatives. Here, we show a possible procedure to select actual threshold values to differentiate between low and high similarities.

In order to define a suitable Pearson correlation threshold value, the biological activity of mitoxantrone was compared to a set of known Top2 poisons and inhibitors (Fig. 3). The threshold of biological activity similarity was set to be at least 0.44 , representing the lowest similarity to mitoxantrone among the annotated Top2 poisons and inhibitors see Additional file 1: Table S1. The procedure to select potential threshold intervals for ROCS and CFP similarities was as follows. First, the percentiles of molecule pairs displaying 0.44 BA similarities were calculated, then structural threshold values corresponding to these percentiles were obtained. For each possible combinations of BA, ROCS and CFP threshold values, the number of putative scaffold hopping molecules was determined. The molecules remain the same when $\mathrm{BA}>=[0.53,0.54]$, ROCS $>=[0.50,0.52]$ and CFP $<[0.32,0.34]$, the final threshold values were hence chosen as $0.54,0.32$ and 0.51 for BA, CFP and ROCS similarities respectively (Additional file 1: Table S2).

\section{In silico docking}

Human topoisomerase IIb in complex with DNA and etoposide, mitoxantrone, ametantrone and amsacrine (3QX3, 4G0V, 4G0W and 4G0U in PDB) was analyzed in docking calculations [23, 24, 55], using the Small-Molecule Drug Discovery Suite 2017-1 (Schrödinger, LLC, New York, NY, 2017) [56]. All four protein structures were prepared using the Protein Preparation Wizard, $\mathrm{H}$-bonds were optimized with the automated procedure. A fifth protein structure was also used during docking calculations: a 4G0U-etopiside complex structure resulting from induced fit docking (IFD) calculations. Ligands were prepared by the Ligprep module with default parameters except that the maximum number of stereoisomers was set to 4. For each target structure, the binding site was defined based on the corresponding drug molecule coordinates. For each ligand, the best docking score was used in the follow up analysis. The decoy library was generated using the DUD-E online tool at http://dude.docking.org/generate [57]. Additionally, '3D similarity decoys' were selected from the DTP agents as molecules, whose 3D shape was similar to a published Top2 ligand [23, 24], but their biological activity based Pearson correlation similarities were low. Conversely, 'Pearson similarity decoys' were selected, whose 3D shape was dissimilar, but their drug toxicity profile was similar to a published Top2 ligand. Docking calculations were performed on all ligand and decoys structures using the Glide SP method (Schrödinger, LLC, New York, NY, 2017) [56]. Figure 6 was created using the PyMOL program. (The PyMOL Molecular Graphics System, Version 1.6, Schrödinger, LLC.)

\section{In vitro decatanation assay}

The inhibitory effect of the compounds on the catalytic activity of Top2 was investigated using the decatenation assay (TopoGEN, Ohio) [58]. $0.2 \mu \mathrm{g}$ catenated kinetoplast DNA (kDNA) was incubated at $37{ }^{\circ} \mathrm{C}$ for $30 \mathrm{~min}$ in the presence of the test compounds and Top2 in a final volume of $20 \mu \mathrm{l}$, containing $50 \mathrm{mM}$ Tris-Cl ( $\mathrm{pH} 8.0), 150 \mathrm{mM} \mathrm{NaCl}, 10 \mathrm{mM} \mathrm{MgCl}_{2}$, $5 \mathrm{mM}$ ATP, $0.5 \mathrm{mM}$ DTT and $30 \mu \mathrm{g} / \mathrm{ml} \mathrm{BSA}$. Mitoxantrone was used as a positive control. The reaction was stopped by a $15 \mathrm{~min}$ incubation at $37^{\circ} \mathrm{C}$ with $3 \mu \mathrm{l}$ SDS containing $1 \mathrm{mg} / \mathrm{ml}$ proteinase $\mathrm{K}$. Samples were 
separated by $1 \%$ agarose gel electrophoresis $(100 \mathrm{~V}$, $30 \mathrm{~min})$. DNA bands were visualized by ethidium bromide. UV-transilluminated gels were documented with the Multi-Analyst software. Dose response curves were fitted to experimental data using the equation $\hat{y}=b+(t-b) \times \log (\text { IC50 })^{n} /\left(\log (\text { conc })^{n}+\log (\text { IC50 })^{n}\right)$ where $b=0, t=100$.

\section{Supplementary information}

Supplementary information accompanies this paper at https://doi. org/10.1186/s13321-019-0390-3.

Additional file 1: Additional text. Additional Text includes the commands used for the ROCS (OpenEye Scientific Software, Santa Fe, NM) and CFP (ChemAxon Ltd., Budapest, Hungary) similarity calculations and Additional Figures and Tables. Figure S1. Flowchart depicting the selection and comparison of DTP molecules used in this study. Figure S2. Histogram of pairwise Pearson correlation values among NSC duplicates (a) and desalted structure duplicates (b). Dashed vertical red line represents the Pearson correlation threshold used in this study to select scaffold-hopping analogues of mitoxantrone, while continuous red vertical line represents the cut-off for keeping duplicate structures. Figure S3. Distribution of the 11,797,653 pairwise similarity values supplemented with the bootstrapped distributions (continuous lines), where available. The vertical lines show the $95 \%$ confidence intervals of the bootstrapped distributions. Figure S4. Additional scaffold hopping candicates of either mitoxantrone, ametantrone, amsacrine or etoposide. Cf. Fig 3 in main text. Figure S5. Example gel photos displaying dose-response Top2 poisoning of NSC637992 and mitoxantrone. Dose response curves were calculated based on the intensities corresponding to the decatenated DNA (red arrow). Figure S6. Scaled density of the docking scores calculated for candidate scaffold hopping analogues of mitoxantrone (blue), the '3D decoy' and the 'biological decoy' sets (orange and red, respectively) and the DUDE-E decoys (grey) when the similarity threshold values were chosen as the strictest (a) and most lenient (b). Figure S7. NSC660839, the '3D decoy' molecule tested in the in vitro decatenation assay. Figure S8. Known Top1 inhibitor scaffolds: camptothecins (NSC94600), indenoisoquinolines (NSC314622), indolocarbazoles. Figure S9. Scaffold hopping candidates of camptothecin obtained using threshold values BA $>0.49$, ROCS $>0.51$, CFP $\leq 0.34$. Figure S10. NSC24818 (podofilox). Figure S11. Scaffold hopping candidates of NSC24818 obtained using threshold values BA $>0.41$, ROCS $>0.48$, CFP $\leq 0.30$. Table $\mathbf{S 1}$. Pearson correlation (BiolAct similarity) of the plC50 values of annotated Top2 poisons to mitoxantrone. Table S2. Selected similarity thresholds to identify putative scaffold hopping analogues of mitoxantrone. Table S3. Docking scores and rankings (from 1173 compounds) of mitoxantrone and its scaffold hopping candidates. Table S4. Maximum of biological, ROCS and 2D similarities compared to mitoxantrone, ametantrone, amsacrine or etoposide. Additionally, docking scores of these compounds. Table $\mathbf{S 5}$. Similarities compared to published Top2 ligands and docking scores of 3D decoy agents. Table S6. Similarities compared to published Top2 ligands and docking scores of biological activity decoy agents. Table S7. Docking scores of DUDE-E decoys.

\section{Acknowledgements}

We thank OpenEye for the use of their softwares OMEGA and ROCS, and ChemAxon for the use of their softwares Standardizer, Structure Checker and ScreenMD. We acknowledge NIIF for awarding us access to resource based in Hungary at Debrecen. The support of Tamás Hornos and Gábor Rőczei from NIIF Institute, Hungary to the technical work is gratefully acknowledged. We thank Gábor Tusnády for granting us access to resource Supermicro computer cluster (256 CPU core, 4Gb RAM/CPU core). Chemical compounds obtained from the NCI DTP are gratefully acknowledged. We thank György Keserü for the critical reading of the manuscript.

\section{Authors' contributions}

AL compiled the GI50 data and wrote the manuscript. AL, DSz and AK conducted similarity calculations. AL and VP identified and categorized scaffold hopping molecules. AL, VP and GSz analyzed and interpreted the results. PH and GSz carried out the in vitro experiments. CsM performed the docking calculations. GSz conceived the project, supervised its evolution and wrote the manuscript. All authors read and approved the final manuscript.

\section{Funding}

A.L. was supported by MTA PD-041/2015 and OTKA PD124467 Grants. C.M. was supported by OTKA K1 15698 grant. G.Sz. was supported by a Momentum Grant from the Hungarian Academy of Sciences (LP-2010-003/2015) and the ERC Starting Grant 260572. Funding from the Austrian Science Fund SFB35 (G.Sz.) is also acknowledged.

\section{Availability of data and materials}

Additional files S1, S2 and S3 are available at https://drive.google.com/drive/ folders/1V8mjEOKNsrOSLIOVaEAgeSgly_wyHyUL?usp=sharing.

\section{Competing interests}

The authors declare that they have no competing interests.

\section{Author details}

${ }^{1}$ Institute of Enzymology, Research Centre for Natural Sciences, Hungarian Academy of Sciences, Budapest 1117, Hungary. ${ }^{2}$ Present Address: Department of Physiology, Semmelweis University, Faculty of Medicine, Budapest 1094, Hungary. ${ }^{3}$ ChemAxon Ltd., Graphisoft park, Záhony u. 7, Budapest 1031, Hungary. ${ }^{4}$ Institute of Cancer Research and Comprehensive Cancer Center, Medical University of Vienna, Borschkegasse 8a, 1090 Vienna, Austria.

Received: 29 May 2019 Accepted: 23 October 2019

Published online: 09 November 2019

\section{References}

1. Seshacharyulu P, Ponnusamy MP, Haridas D, Jain M, Ganti AK, Batra SK (2012) Targeting the EGFR signaling pathway in cancer therapy. Expert Opin Ther Tar 16(1):15-31

2. Swinney D (2013) Phenotypic vs. target-based drug discovery for first-inclass medicines. Clin Pharmacol Ther 93(4):299-301

3. Moffat JG, Rudolph J, Bailey D (2014) Phenotypic screening in cancer drug discovery-past, present and future. Nat Rev Drug Discov 13(8):588-602

4. Cheng T, Li Q, Zhou Z, Wang Y, Bryant SH (2012) Structure-based virtual screening for drug discovery: a problem-centric review. AAPS J 14(1):133-141

5. Geppert H, Vogt M, Bajorath J (2010) Current trends in ligand-based virtual screening: molecular representations, data mining methods, new application areas, and performance evaluation. J Chem Inf Model 50(2):205-216

6. Rester U (2008) From virtuality to reality-virtual screening in lead discovery and lead optimization: a medicinal chemistry perspective. Curr Opin Drug Discov Devel 11(4):559-568

7. Shoemaker $\mathrm{RH}$ (2006) The NCl60 human tumour cell line anticancer drug screen. Nat Rev Cancer 6(10):813-823

8. Weinstein JN, Myers TG, O'connor PM, Friend SH, Fornace AJ, Kohn KW, Fojo T, Bates SE, Rubinstein LV, Anderson NL, Buolamwini JK, vanOsdol WW, Monks AP, Scudiero DA, Sausville EA, Zaharevitz DW, Bunow B, Viswanadhan VN, Johnson GS, Wittes RED, KP (1997) An informationintensive approach to the molecular pharmacology of cancer. Science 275(5298):343-349

9. Paull KD, Shoemaker RH, Hodes L, Monks A, Scudiero DA, Rubinstein L, Plowman J, Boyd MR (1989) Display and analysis of patterns of differential activity of drugs against human tumor cell lines: development of mean graph and COMPARE algorithm. J Natl Cancer Inst 81(14):1088-1092. https://doi.org/10.1093/jnci/81.14.1088

10. Rabow AA, Shoemaker RH, Sausville EA, Covell DG (2002) Mining the National Cancer Institute's Tumor-Screening Database: identification of compounds with similar cellular activities. J Med Chem 45(4):818-840. https://doi.org/10.1021/jm010385b 
11. Wallqvist A, Huang R, Covell DG, Roschke AV, Gelhaus KS, Kirsch IR (2005) Drugs aimed at targeting characteristic karyotypic phenotypes of cancer cells. Mol Cancer Ther 4(10):1559-1568. https://doi. org/10.1158/1535-7163.MCT-05-0224

12. Keskin O, Bahar I, Jernigan R, Beutler J, Shoemaker R, Sausville E, Covell D (2000) Characterization of anticancer agents by their growth inhibitory activity and relationships to mechanism of action and structure. Anticancer Drug Des 15(2):79-98

13. Blower P, Fligner M, Verducci J, Bjoraker J (2002) On combining recursive partitioning and simulated annealing to detect groups of biologically active compounds. J Chem Inf Comput Sci 42(2):393-404. https:// doi.org/10.1021/ci0101049

14. Flower DR (1998) On the properties of bit string-based measures of chemical similarity. J Chem Inf Comput Sci 38(3):379-386

15. Rogers D, Hahn M (2010) Extended-connectivity fingerprints. J Chem Inf Model 50(5):742-754

16. Nicholls A, McGaughey GB, Sheridan RP, Good AC, Warren G, Mathieu M, Muchmore SW, Brown SP, Grant JA, Haigh JA, Nevins N, Jain AN, Kelley B (2010) Molecular shape and medicinal chemistry: a perspective. J Med Chem 53(10):3862-3886. https://doi.org/10.1021/jm900818s

17. Rush TS, Grant JA, Mosyak L, Nicholls A (2005) A shape-based 3-d scaffold hopping method and its application to a bacterial protein-protein interaction. J Med Chem 48(5):1489-1495. https://doi.org/10.1021/ jm0401630

18. Wallqvist A, Huang R, Thanki N, Covell DG (2006) Evaluating chemical structure similarity as an indicator of cellular growth inhibition. J Chem Inf Model 46(1):430-437. https://doi.org/10.1021/ci0501544

19. Sun H, Tawa G, Wallqvist A (2012) Classification of scaffold-hopping approaches. Drug Discov Today 17(7-8):310-324. https://doi. org/10.1016/j.drudis.2011.10.024

20. Hu Y, Stumpfe D, Bajorath J (2016) Recent advances in scaffold hopping: miniperspective. J Med Chem 60(4):1238-1246

21. Skladanowski A, Plisov SY, Konopa J, Larsen AK (1996) Inhibition of DNA topoisomerase II by imidazoacridinones, new antineoplastic agents with strong activity against solid tumors. Mol Pharmacol 49(5):772-780

22. Adams A, Guss JM, Collyer CA, Denny WA, Prakash AS, Wakelin LP (2000) Acridinecarboxamide topoisomerase poisons: structural and kinetic studies of the DNA complexes of 5-substituted 9-amino-( $\mathrm{N}$ (2-dimethylamino) ethyl) acridine-4-carboxamides. Mol Pharmacol 58(3):649-658

23. Wu C-C, Li T-K, Farh L, Lin L-Y, Lin T-S, Yu Y-J, Yen T-J, Chiang C-W, Chan N-L (2011) Structural basis of type II topoisomerase inhibition by the anticancer drug etoposide. Science 333(6041):459-462

24. Wu C-C, Li Y-C, Wang Y-R, LiT-K, Chan N-L (2013) On the structural basis and design guidelines for type II topoisomerase-targeting anticancer drugs. Nucleic Acids Res 41(22):10630-10640. https://doi.org/10.1093/ nar/gkt828

25. Liang H, Wu X, Guziec LJ, Guziec FS, Larson KK, Lang J, Yalowich JC, Hasinoff BB (2006) A structure-based 3d-qsar study of anthrapyrazole analogues of the anticancer agents losoxantrone and piroxantrone. J Chem Inf Model 46(4):1827-1835

26. McGaughey GB, Sheridan RP, Bayly Cl, Culberson JC, Kreatsoulas C, Lindsley S, Maiorov V, Truchon J-F, Cornell WD (2007) Comparison of topological, shape, and docking methods in virtual screening. J Chem Inf Model 47(4):1504-1519. https://doi.org/10.1021/ci700052x

27. Venkatraman V, Peérez-Nueno VI, Mavridis L, Ritchie DW (2010) Comprehensive comparison of ligand-based virtual screening tools against the DUD data set reveals limitations of current 3D methods. J Chem Inf Model 50(12):2079-2093. https://doi.org/10.1021/ci100263p

28. Giganti D, Guillemain H, Spadoni J-L, Nilges M, Zagury J-F, Montes M (2010) Comparative evaluation of 3D virtual ligand screening methods: impact of the molecular alignment on enrichment. J Chem Inf Model 50(6):992-1004. https://doi.org/10.1021/ci900507g

29. Kalászi A, Szisz D, Imre G, Polgár T (2014) Screen3D: a novel fully flexible high-throughput shape-similarity search method. J Chem Inf Model 54(4):1036-1049. https://doi.org/10.1021/ci400620f

30. Szakács G, Annereau J-P, Lababidi S, Shankavaram U, Arciello A, Bussey KJ, Reinhold W, Guo Y, Kruh GD, Reimers M (2004) Predicting drug sensitivity and resistance: profiling $A B C$ transporter genes in cancer cells. Cancer Cell 6(2):129-137
31. Hopkins AL, Keserü GM, Leeson PD, Rees DC, Reynolds CH (2014) The role of ligand efficiency metrics in drug discovery. Nat Rev Drug Discov 13(2):105-121

32. Grisoni F, Merk D, Consonni V, Hiss JA, Tagliabue SG, Todeschini R, Schneider G (2018) Scaffold hopping from natural products to synthetic mimetics by holistic molecular similarity. Commun Chem 1(1):44

33. Pommier $Y$ (2006) Topoisomerase I inhibitors: camptothecins and beyond. Nat Rev Cancer 6(10):789

34. Kaan HYK, Weiss J, Menger D, Ulaganathan V, Tkocz K, Laggner C, Popowycz F, Joseph B, Kozielski F (2011) Structure-activity relationship and multidrug resistance study of new S-trityl-L-cysteine derivatives as inhibitors of Eg5. J Med Chem 54(6):1576-1586

35. Lamb J, Crawford ED, Peck D, Modell JW, Blat IC, Wrobel MJ, Lerner J, Brunet J-P, Subramanian A, Ross KN, Reich M, Hieronymus H, Wei G, Armstrong SA, Haggarty SJ, Clemons PA, Wei R, Carr SA, Lander ES, Golub TR (2006) The Connectivity Map: using gene-expression signatures to connect small molecules, genes, and disease. Science 313(5795):1929-1935

36. Campillos M, Kuhn M, Gavin A-C, Jensen LJ, Bork P (2008) Drug target identification using side-effect similarity. Science 321(5886):263-266. https://doi.org/10.1126/science.1158140

37. Kuhn M, Campillos M, Letunic I, Jensen LJ, Bork P (2010) A side effect resource to capture phenotypic effects of drugs. Mol Syst Biol 6(1):343

38. Lee AY, St.Onge RP, Proctor MJ, Wallace IM, Nile AH, Spagnuolo PA, Jitkova Y, Gronda M, Wu Y, Kim MK, Cheung-Ong K, Torres NP, Spear ED, Han MKL, Schlecht U, Suresh S, Duby G, Heisler LE, Surendra A, Fung E, Urbanus ML, Gebbia M, Lissina E, Miranda M, Chiang JH, Aparicio AM, Zeghouf M, Davis RW, Cherfils J, Boutry M, Kaiser CA, Cummins CL, Trimble WS, Brown GW, Schimmer AD, Bankaitis VA, Nislow C, Bader GD, Giaever G (2014) Mapping the cellular response to small molecules using chemogenomic fitness signatures. Science 344(6180):208-211

39. Yang H, Sun L, Wang Z, Li W, Liu G, Tang Y (2018) Admetopt: a web server for admet optimization in drug design via scaffold hopping. J Chem Inf Model 58:2051-2056

40. screenmd (2014) ChemAxon Ltd., JChem6.3. http://www.chemaxon.com

41. screen3d (2014) ChemAxon Ltd., JChem6.3. http://www.chemaxon.com

42. Jenkins JL, Glick M, Davies JW (2004) A 3d similarity method for scaffold hopping from known drugs or natural ligands to new chemotypes. J Med Chem 47(25):6144-6159

43. Zhang Q, Muegge I (2006) Scaffold hopping through virtual screening using $2 \mathrm{~d}$ and $3 \mathrm{~d}$ similarity descriptors: ranking, voting, and consensus scoring. J Med Chem 49(5):1536-1548

44. Fourches D, Muratov E, Tropsha A (2010) Trust, but verify: on the importance of chemical structure curation in cheminformatics and qsar modeling research. J Chem Inf Model 50(7):1189-1204

45. Fourches D, Muratov E, Tropsha A (2016) Trust, but verify Il: a practical guide to chemogenomics data curation. J Chem Inf Model 56(7):1243-1252

46. Shankavaram UT, Reinhold WC, Nishizuka S, Major S, Morita D, Chary KK, Reimers MA, Scherf U, Kahn A, Dolginow D, Cossman J, Kaldjian EP, Scudiero DA, Petricoin E, Liotta L, Lee JK, Weinstein JN (2007) Transcript and protein expression profiles of the $\mathrm{NCl}-60$ cancer cell panel: an integromic microarray study. Mol Cancer Ther 6(3):820-832. https://doi. org/10.1158/1535-7163.MCT-06-0650

47. Reinhold WC, Sunshine M, Liu H, Varma S, Kohn KW, Morris J, Doroshow J, Pommier Y (2012) Cell miner: a web-based suite of genomic and pharmacologic tools to explore transcript and drug patterns in the NCI-60 cell line set. Cancer Res 72(14):3499-3511. https://doi.org/10.1158/00085472.CAN-12-1370

48. standardizer (2014) ChemAxon Ltd., JChem6.3. http://www.chemaxon. com

49. structurechecker (2014) ChemAxon Ltd., JChem6.3. http://www.chema xon.com

50. Hawkins PCD, Skillman AG, Warren GL, Ellingson BA, Stahl MT. OMEGA 2.5.1.4. OpenEye Scientific Software, Santa Fe, NM. http://www.eyesopen. com

51. Instant JChem (2014) ChemAxon Ltd., Instant JChem6.3. http://www. chemaxon.com

52. Hawkins PC, Skillman AG, Nicholls A (2007) Comparison of shape-matching and docking as virtual screening tools. J Med Chem 50(1):74-82

53. ROCS 3.2.0.4. OpenEye Scientific Software, Santa Fe, NM. http://www. eyesopen.com 
54. Hawkins PC, Skillman AG, Warren GL, Ellingson BA, Stahl MT (2010) Conformer generation with OMEGA: algorithm and validation using high quality structures from the Protein Databank and Cambridge Structural Database. J Chem Inf Model 50(4):572-584

55. Berman HM, Westbrook J, Feng Z, Gilliland G, Bhat TN, Weissig H, Shindyalov IN, Bourne PE (2000) The protein data bank. Nucleic Acids Res 28(1):235-242

56. Small-Molecule Drug Discovery Suite 2017-1: Glide (2017) version 6.4. Schrödinger, LLC, New York

57. Mysinger MM, Carchia M, Irwin JJ, Shoichet BK (2012) Directory of usefu decoys, enhanced (DUD-E): better ligands and decoys for better benchmarking. J Med Chem 55(14):6582-6594

58. Dornetshuber R, Heffeter P, Lemmens-Gruber R, Elbling L, Marko D, Micksche M, Berger W (2009) Oxidative stress and DNA interactions are not involved in Enniatin- and Beauvericin-mediated apoptosis induction. Mol Nutr Food Res 53(9):1112-1122
59. Weinstein JN, Kohn KW, Grever MR, Viswanadhan VN, Rubinstein LV, Monks AP, Scudiero DA, Welch L, Koutsoukos AD, Chiausa AJD, KP (1992) Neural computing in cancer drug development: predicting mechanism of action. Science 258(5081):447-451. https://doi.org/10.1126/scien ce. 1411538

\section{Publisher's Note}

Springer Nature remains neutral with regard to jurisdictional claims in published maps and institutional affiliations.
Ready to submit your research? Choose BMC and benefit from:

- fast, convenient online submission

- thorough peer review by experienced researchers in your field

- rapid publication on acceptance

- support for research data, including large and complex data types

- gold Open Access which fosters wider collaboration and increased citations

- maximum visibility for your research: over 100M website views per year

At BMC, research is always in progress.

Learn more biomedcentral.com/submissions 